\title{
Advanced Services for Supply Chain Design Processes in Collaborative Networks
}

\author{
Eva A. Coscia ${ }^{1}$, Rosanna Fornasiero ${ }^{2}$, João Bastos ${ }^{3}$, Américo Azevedo ${ }^{3}$, \\ Domenico Rotondi ${ }^{4}$, and Salvatore Piccione ${ }^{4}$ \\ ${ }^{1}$ TXT e-Solutions SPA, Via Frigia 27, 20126 Milano, Italy \\ ${ }^{2}$ ITIA-CNR, Via Bassini 15, 20133 Milano, Italy \\ ${ }^{3}$ INESC Porto, Campus da FEUP, Rua Dr Roberto Frias 378, 4200-465 Porto, Portugal \\ ${ }^{4}$ TXT e-solutions SpA, c/o Tecnopolis N.O. Str. Prov. Per Casamassima Km 3, \\ 70010 Valenzano (BA), Italy
}

\begin{abstract}
Design and production of small series for specific customer target groups in collaborative networks can help companies to increase their competitiveness. This paper aims to describe a new framework of services for collaborative networking for on-demand productions. After describing the business processes that need to be supported by innovative tools for partner search and collaborative production planning, we describe the developed services and how they are integrated to ease the communication between different activities.
\end{abstract}

Keywords: Partner Search, Collaborative Networks, Value Chain, Business Intelligence, Collaborative Framework.

\section{Introduction}

In recent years, the importance of collaborative strategies addressing the small series production of highly-customized products is emerging at industrial level. European companies of the Textile, Clothing and Footwear industry (TCFI) are asked to supply small series of innovative and fashionable goods having high quality, affordable prices and eco-compatibility. In order to support the design and production of such kind of products, companies need to be fast and flexible in answering to market demand. The CoReNet ${ }^{1}$ Project provides a set of tools and services addressing those requirements.

This paper, in particular, presents some of the services developed in the CoReNet project that address the complexity of collaborative networks management able to respond to the high variability of the consumers demand and expectations. The particular case of TCFI companies producing healthy and fashionable products is taken as case study.

\footnotetext{
${ }^{1}$ CoReNet (Customer-ORiented and Eco-friendly NETworks for healthy fashionable goods: www.corenet-project.eu)
} 
Small series and customized products require totally different supply network management, where each company should be able to collaborate with specialized partners. In customized production, supply networks should be easily re-configurable for each covered market niche, or, even, for each customer order. In practice these scenarios are characterized by a very large number of small orders, each of them involving different partner companies, selected on the basis of their availability and capabilities. In this context, partner profiling and monitoring, as well as co-planning and control processes need to be re-engineered and supported by integrated services and based on easy to retrieve, easy to manage and reliable information.

After a short description of the business processes that are required and supported by the developed tools, the paper will shortly describe the tools and how they are integrated for a combined service to networked companies.

\section{Collaborative Business Processes for Supply Chain Design}

Supply networks should be easily reconfigured time after time according to the specific orders as expressed by target groups, for the creation of dedicated small series, or by single people, in case of customized products. Two business processes have been taken into account to support TCFI companies in small series production: Partner Profiling and Search and Collaborative Planning.

These processes start at the end of the design and development process, when all the items belonging to a specific collection and the related basic elements have been defined (e.g.: 2D/3D models, part programs for cutting, printing and any other automation step) and the products need to be prepared for sales. At this stage TCFI companies need to manage many different product variants as well as the design and production of related components.

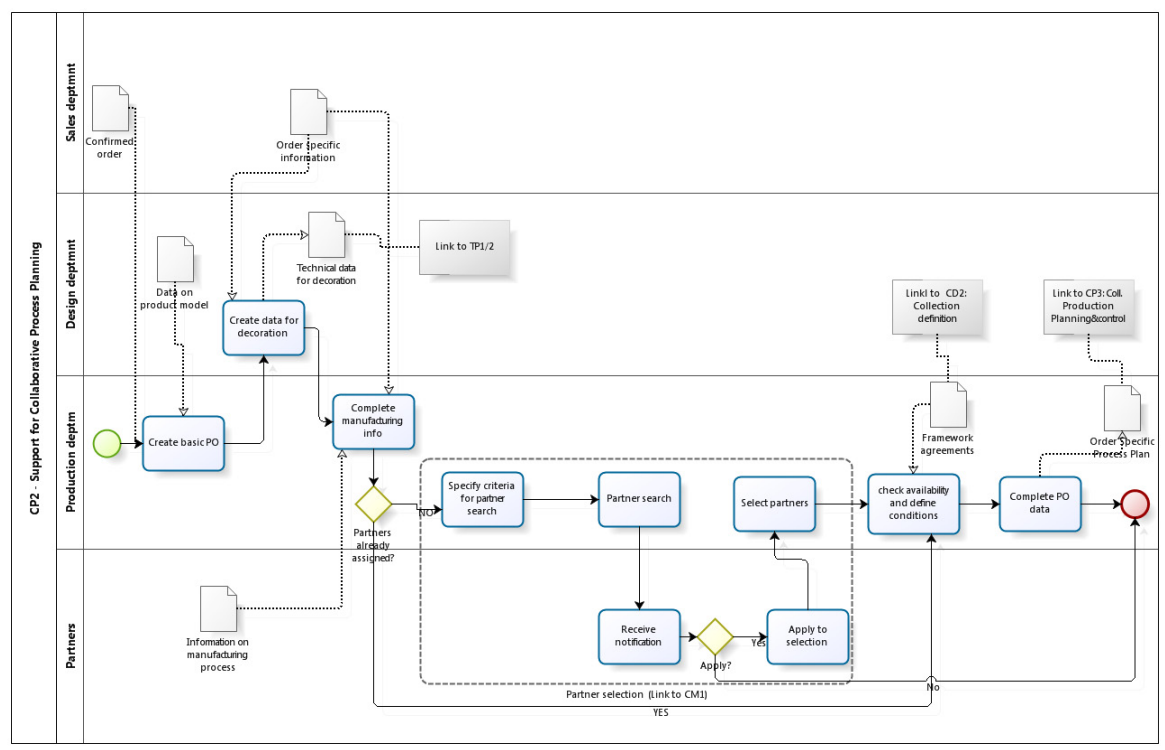

Fig. 1. Collaborative Process planning diagram 
Beside the early phases for the design and development of the collection, once the sales start and the actual product request is defined and configured, in terms of measures, quantities and due dates, all the complete set of information required for the actual production can be finally defined (final BOM, accessories, etc.) and the production orders (PO) can be generated for a specific customer order. It is therefore possible to design and create a specific Supply Chain for the processing of the customer order, which implies to have identified all the suppliers and partners in charge of carrying out all the external activities, assigned the whole set of operations associated to a specific product and finally obtained all the information and data needed for the planning activities.

The business processes taken into consideration are depicted by BPMN diagrams and some activities are represented in Figure 1.

The process of Partner Search for supplying components and for outsourcing operations may occur both at strategic and operational level.

Many different approaches are proposed in literature on this issue and inspiration is taken from (Lambert, et al. 2001) for the application of methodologies to measure performance as a way to improve collaborative networks, from (Gunasekaran, et al. 2005) for the KPIs used as criteria to suggest supply network partners, from (Jarimo, et al. 2006) and (Crispim, et al. 2007) who introduced other criteria, like trustworthiness and risk values and from (Westphal, et al. 2007) for the utilization of KPIs related to collaboration and commitment.

During the definition of the product collection, the manufacturer needs to strategically identify partners who can support both the design and the production of the collection itself or the product customization. Strategic identification of partners is based on a partners profiling process: manufacturer assigns to each partner category some indicators based on previous performance and selects them specifying some selection criteria. From the organizational point of view, the relationship to be established is not only a pure buyer-seller relationship, but it is a more complex relationship that may require sharing data both on product quantity/quality, as well as on the company performances, machines, capacity availability, environmental performance, etc.

Once a customer order is collected, it is necessary to choose among the preselected partners those that will be activated for that specific order. In order to shorten the Partner search process at operational level the search is based on capacity availability for the lead time required by the manufacturer where previous agreements on quality and costs have already been taken. At this level it is mainly a matter of monitoring status of suppliers and outsourcers' availability.

The Partner search tool developed in CoReNet is based on the definition of Partner Profiles including both data provided by the supplier itself (e.g. administrative data, description of competences, provided material or process, etc.), as well as data derived from the analysis of the suppliers' past behavior based on performance indicators like the following ones: 
- Collaboration degree: indicating how the supplier behaved in previous collaborations (e.g.: number of collaborations held in the previous period, number of successful negotiations, etc.).

- Products quality: reflecting the quality of the provided products (e.g.: number of defective products, etc.).

- Flexibility: describing the partner's ability to react rapidly and adapt to changes in the order or at production time.

Subsequently collaborative planning process manages the activities to support companies' plans towards each other to reach a joint optimization of the planning across departmental boundaries. Collaborative planning involves activities by means of which individuals coordinate their planning processes (Windischer, Grote et al. 2009).

When working with actual and complex business scenarios (like the textile or footwear sectors, for instance), local autonomy of each entity is an important issue.

For this reason, based on the analysis of the state-of-the-art, an innovative collaborative planning concept and approach is proposed in CoReNet for supporting decision making in supply network planning, respecting the requirements of complex products and small series production networks. The new approach is based on decentralized and cooperative actions and offers user friendly interface to the supply network stakeholders, supporting complex negotiation practices on a web-based platform. Furthermore through the use of a multi-criteria analysis, it is possible to define assessment mechanisms in order to optimize the overall supply network planning process

This approach is based on a decentralized negotiation model, which allows partners to propose new delivery dates and costs, represented graphically in Figure 2.

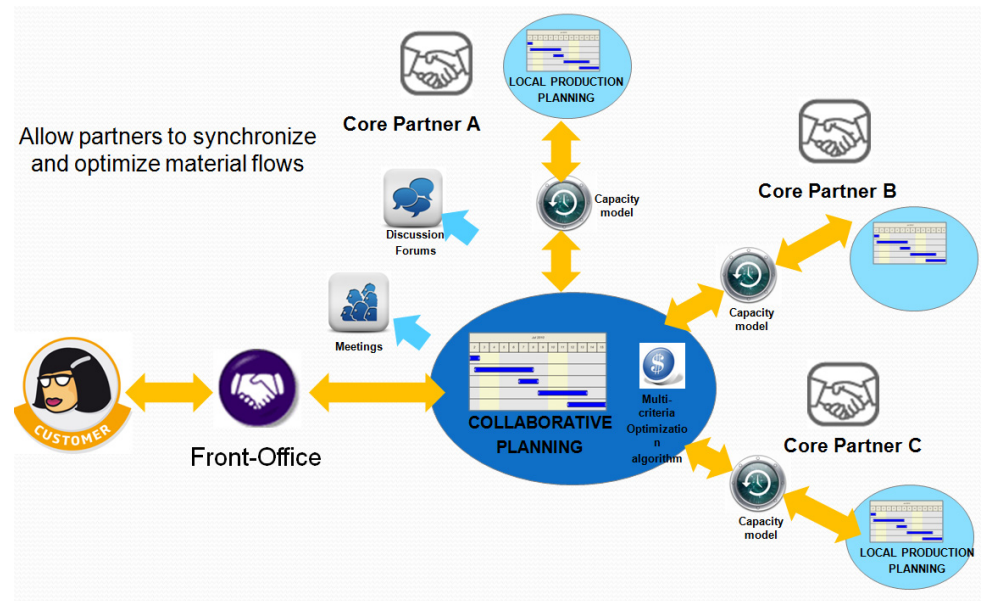

Fig. 2. Collaborative planning approach 


\section{The Services Supporting the Business Processes}

\subsection{The Partner Search Module}

The Partner Search (PS) service offers partner profiling and searching capabilities and is one of the key elements of the CoReNet architecture to set-up supply chains. It is a web-based tool that allows a manufacturer to manage knowledge about its suppliers, by creating and updating Partners Profiles (a set of parameters describing competences and past performance), by visualizing data and performance indicators and by providing search features to select those partners that best match the entered criteria.

The Partner Search service provides an AJAX graphical user interface and a REST API. Through this REST interface, information is made available as two different, but equivalent, output formats: RDFa enriched XHTML, so that the service is accessible using a standard web browser, or RDF enriched XML, that is more suitable for automatic processing by external applications. The REST interface allows integrating the PS tool with other CoReNet tools in order to set up powerful services for the supply chain design and for the automatic detection of suppliers that best fit a set of search criteria.

Data can be both manually entered by human users, (through a GUI implemented as a portlet) and automatically retrieved from legacy systems (e.g. manufacturer's ERP systems).

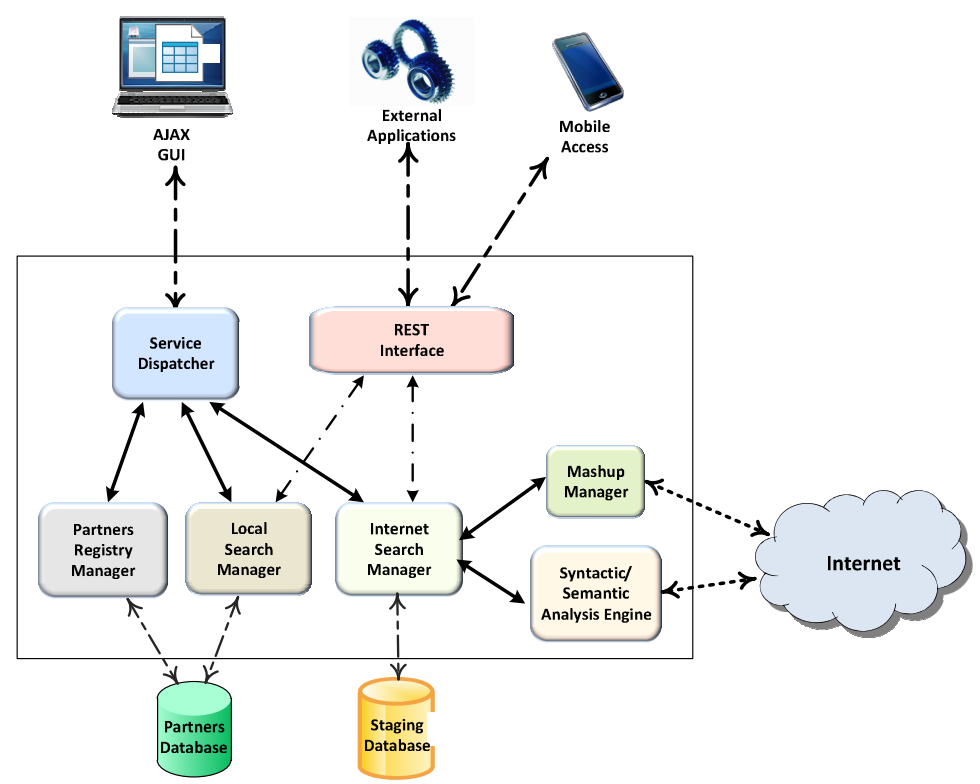

Fig. 3. Partner search module structure 
The system consists of three main blocks:

- Partner Registry Manager: this functional block is in charge of providing the functionalities to add new, or revise existing, partners.

- Local Search Manager: this block provides all search capabilities on profiles of partners with which the manufacturer has a past work history and for which, as a consequence, has KPIs. This module provides its functionalities both via the GUI and via the REST API;

- Internet Search Manager: this block, finally, is in charge of performing searches for potential partners on a set of Internet sites.

\subsection{Workflow Manager}

A Workflow Management (WfM) solution should guarantee companies to create network flows and communicate with partners more efficiently and in an intuitive way. These issues are extremely important especially in the case of small series production, where the exchange of orders and business documents usually happens more frequently than for normal production and the time for producing and exchanging them might be very strict. Therefore it is necessary to have agile tools and services that allow SMEs to quickly react to orders and setup production.

In this context, it is very useful to define a set of basic workflow templates because small series orders come very frequently and are very similar to each other. Such templates might be directly deployed with no need of further adjustments.

The WfM architecture is actually based on an Enterprise Service Bus (ESB) that takes care of: managing the exchange of information with external applications/services (ERP, CRM, etc.), decoupling the specific way the information is transferred from an external application/service to the ESB and vice versa (the WfM offers transport protocols abstraction by using multiple binding components that provides in/out interoperability with the outside world), as well as routing the received information according to a set of rules or information. The CoReNet WfM platform supports the deployment and the execution of workflows using Enterprise Integration Patterns (EIP - see http://www.eaipatterns.com/) and provides a UBL Business Rules Execution Engine: both technologies are used to manage business documents exchange within a specific business process context.

\subsection{Collaborative Planning Module}

The Collaborative Planning (CP) module is a web-based tool deployed under the Liferay portlet container platform. It is a portlet that offers different web-based views accessible for specific user groups/roles supporting user interaction through the planning tool as depicted in the picture 4 . 


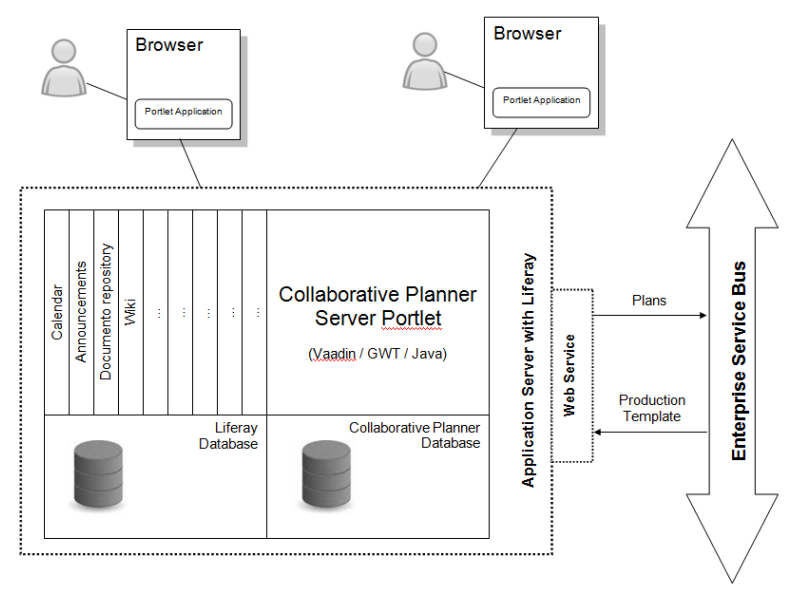

Fig. 4. Module architecture diagram for the Collaborative Planning module

The CP module allows each partner to directly propose new delivery dates, lead times and costs, via a web-based planning graphical tool which is available and shared by all supply network partners. Every time a partner proposes a change on a given operation, it is (actually) asking the affected partner to accept this change (and declare its cost) or to make a counter-proposal. Each negotiation round correspond to a pre-defined time period available to discuss/negotiate delivery times and costs, allowing partners to present quotations for each request-for-quotation (RfQ) performed by other partners.

Each proposed change (which "triggers" RfQs to all involved partners), actually asks the partners to present quotations, which might totally or partially meet, the asked RfQ or even suggest new changes. When a proposal has $100 \%$ agreement of all partners (i.e. "no pending notifications") it will be considered a plan; although it might be changed by any supply network partner, as long as the negotiation time period is not expired. This plan is serialized according to a set of criteria defined in advance by the Front-office and Core partners. For each of these criteria, it is possible to define a degree of importance, using a percentage score as a final weighting factor, which will used to calculate the best partner proposal. In this way each criterion has not got the same importance but each one has got an importance expressed by a ranking system.

The CP promotes a decentralized approach on the definition of the supply networking planning, since every Core partner has the same "weight" on the decision process as it is capable of proposing and participating actively on the characterization of each required operation and to request from any other partner a change on the plan. Therefore, the proposed planning approach integrated with the partner profiling and search service fits the needs of customer-oriented supply networks in achieving flexibility and responsiveness to the market demands. 


\section{Integration of the Services and Common Visualization Layer}

The ability of TCFI industries to quickly adapt to new orders and to consumers' requests is the key factor in the design and set-up of supply chain for the production of small series or even single customized items. Therefore a flexible integration of services aimed at rapidly and accurately identifying partners, at communicating with them, and collaboratively agreeing on production plans would provide a great benefit on the supported business process.

Before defining a production plan, a manufacturer should identify the best candidates to become suppliers. Therefore the CP calls the PS through the REST API, providing a list of criteria and KPIs that better define the potential partners for fulfilling the small-series order.

The PS returns an XML document that contains the list of candidates that best match the searching criteria. The same REST API allows also retrieving complete profiles of the selected partners. When the CP has identified the best candidates for setting up the supply chain, some business documents are exchanged between the manufacturer and the (potential) suppliers, such as RfQs, Bills of Material (BOM) etc.

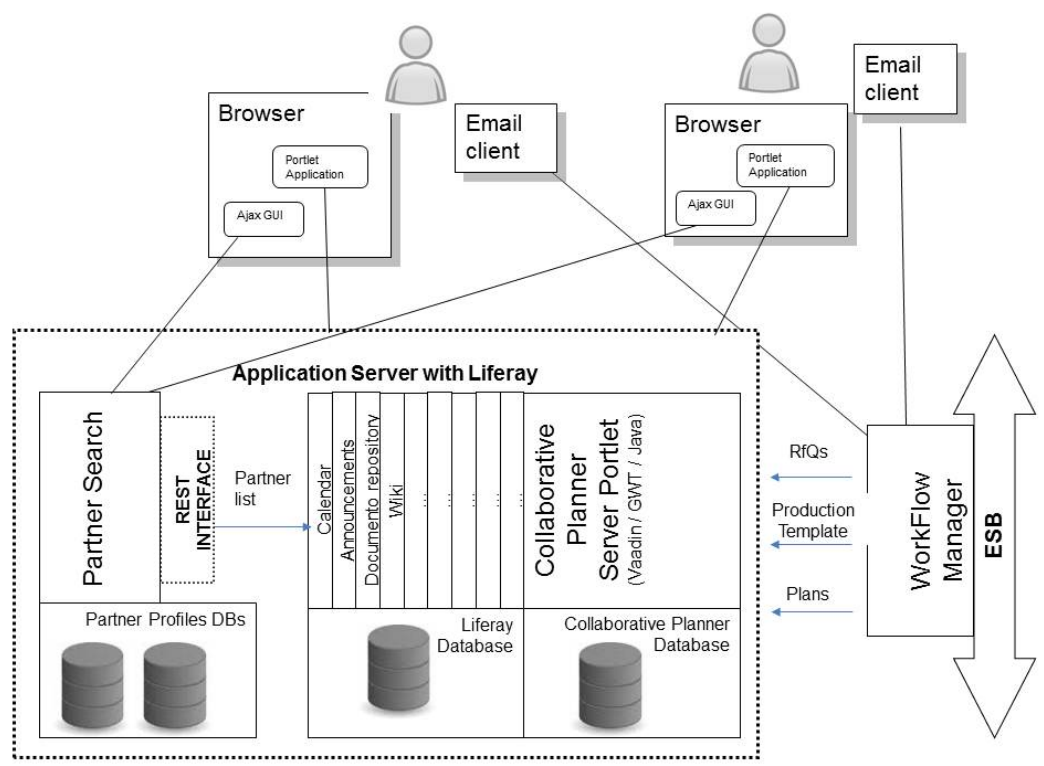

Fig. 5. The integration among tools for supply chain design and set-up

The management of this complex flow of information is performed through the WfM tool, which is activated by the $\mathrm{CP}$ in order to dispatch documents to the right parties and to check their contents and consequently apply business rules. These rules can vary from the mere check of the document structure, to more refined checks on the actual content of the document that trigger pre-defined actions (e.g.: if some 
values of a RfQ do not respect the defined rules, the RfQ is refused and its owner is notified about that).

The figure 5 represents the technical choices performed for integrating the CoReNet tools developed to support the Supply Chain design and set-up.

From a technical point of view, the integration between the CP and the WfM is performed by exploiting the SMTP binding component offered by the ESB underlying the WfM tool. Therefore the two tools exchange emails containing business documents on which pre-defined business rules can be applied.

The applications are made available to the end users through a unique access point, based on the Liferay portal (called the CoReNet Collaboration Portal) that enriches these services with social networking functionalities and advanced communication services for commenting, ranking, reporting bugs and asking for assistance.

The proposed approach provides several benefits for manufacturers and suppliers of the TCFI sector that are looking for agile solutions for the order management and the production plan processes supporting the production of small series. Indeed, the solution:

- $\quad$ is easily accessible and easy to use, as the tools provide advanced GUI and are available within a unique portal (thus no installation is required);

- $\quad$ supports the exchange and the automatic check of business information through well-known channels, like the emails (hiding technical details about the internal format of the exchanged documents);

- helps the selection of partners leveraging on information already owned by the manufacturer and provides an open collaborative environment where planning with the selected ones an agreed production plan.

\section{$5 \quad$ Conclusions and Further Research}

In this research, it has been studied a conceptual approach for managing supply chain set up process in collaborative networks, based on a set of services that address the network definition (partner profiling and search) and the network operation. This innovative approach presents a truthful framework for partner selection and subsequent collaborative negotiation among partners in the TCFI supply networks, providing support for the definition and organization of operations and activities relevant to fulfill customer orders of high customizable products and services.

The increase of the collaborative level in business processes is not only a matter of changing the organization of the related activities but of creating bridges among them and among involved actors. ICT technologies can play a relevant role for the improvement of business processes performance. Moreover the service orientation of the nowadays technologies allows networks of companies to deal with the complexity of fast changing customer demand and need of flexibility using IT applications which allows to collect and manage easily updated information on suppliers, products, time scheduling, etc. The innovative aspect of this work is linked to the definition of collaborative paths between suppliers and manufacturers and supporting them with 
services that cover different activities to exchange information and data for the configuration of collaborative networks. Nevertheless service orientation allows integrating and coordinating distribute ICT modules which can be complementary and can easily exchange information and data.

The developed services and related integration protocols are under validation with some companies from the TCFI and further development are planned to make the services fully compliant with their requirements.

Acknowledgments. The research leading to these results has received funding from the European Union's Seventh Framework Programme (FP7/2007-2013) under grant agreement $n^{\circ}[260169]$.

\section{References}

1. Crispim, J.A., de Sousa, J.P.: Multiple Criteria Partner Selection in Virtual Enterprises. In: Camarinha-Matos, L., Afsarmanesh, H., Novais, P., Analide, C. (eds.) Establishing The Foundation Of Collaborative Networks. IFIP AICT, vol. 243, pp. 197-206. Springer, Boston (2007)

2. Gunasekaran, A., Williams, H.J., Mcgaughey, R.E.: Performance measurement and costing system in new enterprise. Technovation 25(5), 523-533 (2005)

3. Jarimo, T., Salkari, I., Bollhalter, S.: Partner Selection with Network Interdependencies: An Application. In: Camarinlia-Matos, L., Afsarmanesh, H., OUus, M. (eds.) Network-Centric Collaboration and Supporting Fireworks. IFIP AICT, vol. 224, pp. 389-396. Springer, Boston (2006)

4. Lambert, D.M., Pohlen, T.L.: Supply Chain Metrics. International Journal of Logistics Management 12(1), 1-19 (2001)

5. Westphal, I., Thoben, K.D., Seifert, M.: Measuring Collaboration Performance in Virtual Organizations. In: Camarinha-Matos, L., Afsarmanesh, H., Novais, P., Analide, C. (eds.) Establishing the Foundation of Collaborative Networks. IFIP AICT, vol. 243, pp. 33-42. Springer, Boston (2007)

6. Windischer, A., Grote, G., et al.: Characteristics and organizational constraints of collaborative planning. Cognition, Technology \& Work 11(2), 87-101 (2009) 